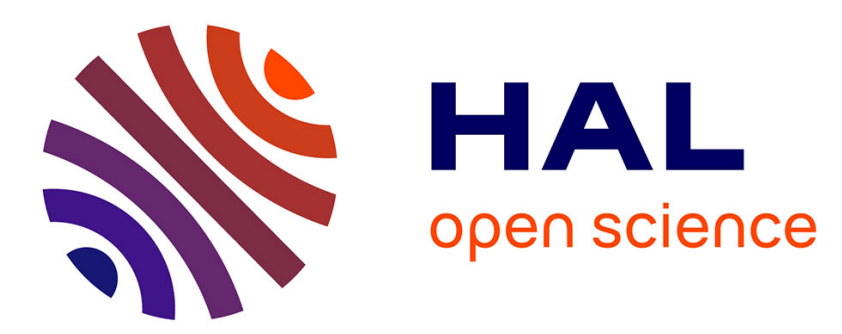

\title{
Sensitivity and optimal forcing response in separated boundary layer flows
}

Frédéric Alizard, Stefania Cherubini, Jean-Christophe Robinet

\section{To cite this version:}

Frédéric Alizard, Stefania Cherubini, Jean-Christophe Robinet. Sensitivity and optimal forcing response in separated boundary layer flows. Physics of Fluids, 2009, 21 (064108), pp.3. 10.1063/1.3153908 . hal-00799747

\section{HAL Id: hal-00799747 https://hal.science/hal-00799747}

Submitted on 13 Mar 2013

HAL is a multi-disciplinary open access archive for the deposit and dissemination of scientific research documents, whether they are published or not. The documents may come from teaching and research institutions in France or abroad, or from public or private research centers.
L'archive ouverte pluridisciplinaire HAL, est destinée au dépôt et à la diffusion de documents scientifiques de niveau recherche, publiés ou non, émanant des établissements d'enseignement et de recherche français ou étrangers, des laboratoires publics ou privés. 


\section{AIP Fluids}

\section{Sensitivity and optimal forcing response in separated boundary layer flows}

Frédéric Alizard, Stefania Cherubini, and Jean-Christophe Robinet

Citation: Phys. Fluids 21, 064108 (2009); doi: 10.1063/1.3153908

View online: http://dx.doi.org/10.1063/1.3153908

View Table of Contents: http://pof.aip.org/resource/1/PHFLE6/v21/i6

Published by the American Institute of Physics.

\section{Related Articles}

Instability of a backward-facing step flow modified by stationary streaky structures

Phys. Fluids 24, 104104 (2012)

Dynamics of magnetic chains in a shear flow under the influence of a uniform magnetic field

Phys. Fluids 24, 042001 (2012)

Boundary layer development in the flow field between a rotating and a stationary disk

Phys. Fluids 24, 033601 (2012)

Slip boundary for fluid flow at rough solid surfaces

Appl. Phys. Lett. 100, 074102 (2012)

Wang's shrinking cylinder problem with suction near a stagnation point

Phys. Fluids 23, 083102 (2011)

\section{Additional information on Phys. Fluids}

Journal Homepage: http://pof.aip.org/

Journal Information: http://pof.aip.org/about/about_the_journal

Top downloads: http://pof.aip.org/features/most_downloaded

Information for Authors: http://pof.aip.org/authors

\section{ADVERTISEMENT}

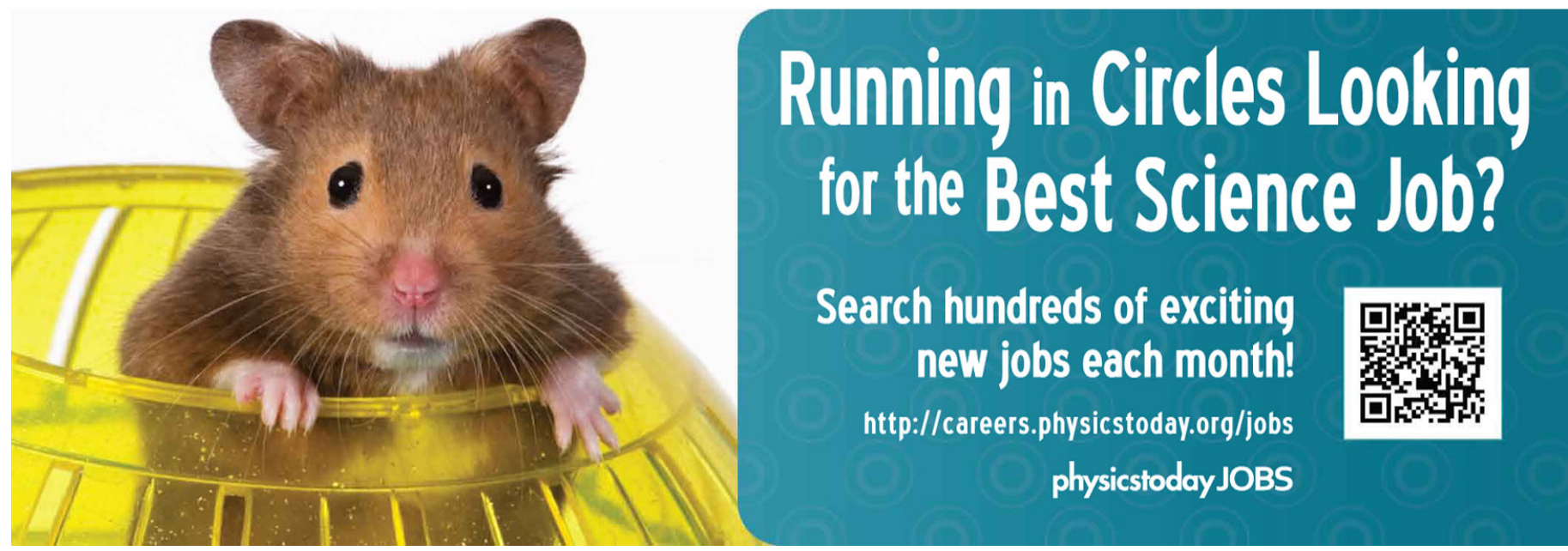




\title{
Sensitivity and optimal forcing response in separated boundary layer flows
}

\author{
Frédéric Alizard, ${ }^{\text {a) }}$ Stefania Cherubini, ${ }^{\text {b) }}$ and Jean-Christophe Robinet \\ SINUMEF Laboratory, Arts and Métiers, ParisTech, 151 Bd. de l'Hôpital, 75013 Paris, France
}

(Received 16 June 2008; accepted 8 May 2009; published online 26 June 2009)

The optimal asymptotic response to time harmonic forcing of a convectively unstable two-dimensional separated boundary layer on a flat plate is numerically revisited from a global point of view. By expanding the flow disturbance variables and the forcing term as a summation of temporal modes, the linear convective instability mechanism associated with the response leading to the maximum gain in energy is theoretically investigated. Such a response is driven by a pseudoresonance of temporal modes due to the non-normality of the underlying linearized evolution operator. In particular, the considered expansion on a limited number of modes is found able to accurately simulate the linear instability mechanism, as suggested by a comparison between the global linear stability analysis and a linearized direct numerical simulation. Furthermore, the dependence of such a mechanism on the Reynolds number and the adverse pressure gradient is investigated, outlining a physical description of the destabilization of the flow induced by the rolling up of the shear layer. Therefore, the convective character of the problem suggests that the considered flat plate separated flows may act as a selective noise amplifier. In order to verify such a possibility, the responses of the flow to the optimal forcing and to a small level of noise are compared, and their connection to the onset of self-excited vortices observed in literature is investigated. For that purpose, a nonlinear direct numerical simulation is performed, which is initialized by a random noise superposed to the base flow at the inflow boundary points. The band of excited frequencies as well as the associated peak match with the ones computed by the asymptotic global analysis. Finally, the connection between the onset of unsteadiness and the optimal response is further supported by a comparison between the optimal circular frequency and a typical Strouhal number predicted by numerical simulations of previous authors in similar cases. (C) 2009 American Institute of Physics.

[DOI: $10.1063 / 1.3153908]$

\section{INTRODUCTION}

When a laminar boundary layer encounters a sufficiently large adverse pressure gradient, a laminar separation bubble (referenced as LSB hereafter) occurs. Many engineering applications such as low Reynolds number aerodynamics configurations of airfoils or car industries and turbomachineries involve typical structures of LSB. Since the first observations of Jones, ${ }^{1}$ flow separation was extensively studied, experimentally as well as numerically. A part of these prior researches deals with its time-averaged mean and steady structure such as the experimental work of Gaster, ${ }^{2}$ the first numerical attempt of Briley, ${ }^{3}$ and the method of matched asymptotic expansions provided by the triple deck theory. ${ }^{4}$

A typical feature of LSB is its very unstable nature and its high sensitivity to background disturbances, even at low Reynolds numbers. This property is often synonymous of loss of aerodynamic performances such as increase in the drag or loss of lift on airfoils at angle of attack close to static stall values. As a consequence, many investigations have been carried out on the onset of unsteadiness in several configurations; for instance, the flat plate separated boundary layer has been studied by means of direct numerical simulations (referenced as DNSs hereafter) ${ }^{5,6}$ and experiments, ${ }^{7}$ as

\footnotetext{
${ }^{a)}$ Author to whom correspondence should be addressed. Electronic mail: frederic.alizard@paris.ensam.fr.

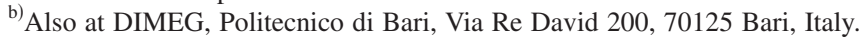

well as the backward facing step flow (see Refs. 8 and 9 for instance). In particular, the self-sustained oscillatory behavior as well as the role of topological changes in the separated flows has received a lot of attention during the last two decades. More specifically, it is now well established that the onset of global instabilities observed in absence of permanent external perturbations is related to the existence of a local absolute instability. ${ }^{10}$ In that respect, the nonlinear selection criteria based on local properties of the flow used by Marquillie and Ehrenstein ${ }^{11}$ was allowed to explain the global high frequency unsteadiness of a recirculation bubble confined between two bumps. This global instability mechanism is characterized by a resonator dynamics similar to the one observed in many open shear flows, for instance, the self-sustained oscillations occurring in the wake of a cylinder. ${ }^{12}$ In such cases, the associated space-time dynamics of the flow is intimately connected to the existence of an unstable global mode. A resonator dynamics driven by a three-dimensional mechanism was observed in a flat plate separated flow by Theofilis et al., ${ }^{13}$ in a flow over a backward facing step by Barkley et al. ${ }^{14}$ or behind a bump by Gallaire $e t a l .{ }^{15}$ The same authors identified a slowly amplified, large scale, stationary, and unstable global mode, not revealed by a local instability analysis, which is able to induce a topological change in the flow.

Nevertheless, some authors recently conjectured that the occurrence of self-sustained oscillations in separated flows 
observed numerically or experimentally may be attributed not only to a resonator dynamics, but should also take into account the influence of external perturbations, as discretization errors in numerical simulations or environment noise occurring in experiments. For instance, Kaiktsis et al. ${ }^{9}$ reported discrepancies among various numerical simulations of the time asymptotic state of a two-dimensional (2D) flow over a backward facing step. Typically, the onset of global unsteadiness appears to be closely dependent on the numerical method and the grid resolution and well below the emergence of an unstable global mode, as reported by Barkley et al. ${ }^{14}$ The response of the convective modes to the level of background noise due to the discretization errors is thus proposed by Kaiktsis et al. ${ }^{9}$ to explain such discrepancies in the asymptotic regime. A similar convective mechanism sustained by the presence of numerical noise is proposed by Wasistho et al. ${ }^{16}$ as an explanation of the onset of unsteadiness in a flat plate separated flow. All these studies suggest that an inherent random background noise may generate unsteadiness in a separated flow. Therefore, the amplifier character of the flow which is due to the presence of a convectively unstable region may play a major role in the capability of the flow to self-sustain perturbations. Nevertheless, the onset of unsteadiness and selected frequencies are still an open question at which no clear answer is as far as we know provided.

In the present contribution, we reassess the selective noise amplifier dynamics of a typical flat plate separated flow by means of a so-called global linear stability approach based on $2 \mathrm{D}$ temporal eigenmodes. ${ }^{17}$ In particular, the convective instability mechanism underlying the global optimal response to a harmonic forcing and its associated energy gain in the asymptotic regime is investigated. It is worth to notice that such a methodology is relevant in the configuration here considered, where nonparallel effects are not negligible. Since the seminal work of Cossu and Chomaz ${ }^{18} 10$ years ago on the space-time dynamics of open flows, and the review of Chomaz $^{19}$ in 2005, it is well known that a global amplifier dynamics could derive from the convective instabilities due to the nonorthogonality of the set of global eigenmodes associated with the considered flow. Since then, this topic has received the attention of several researchers, turning into a vivid research field. In particular, in the previous works of Ehrenstein and Gallaire, ${ }^{20}$ Alizard and Robinet, ${ }^{21}$ and Åkervik et $a l^{22}$ on a flat plate boundary layer, a large transient growth has been observed due to the optimal nonmodal amplification of a localized perturbation. An appropriate superposition of global eigenmodes has shown that the optimal perturbation takes the form of a wave packet traveling along the flat plate and amplifying itself, leading to an increase in the kinetic energy of the perturbation.

Furthermore, similar global stability analysis have been performed in various configurations as a falling liquid curtain by Schmid and Henningson, ${ }^{23}$ an open cavity flow by Åkervik et $a l .{ }^{24}$ and Henningson and Åkervik, ${ }^{25}$ or a flow behind a bump by Ehrenstein and Gallaire ${ }^{26}$ and pointed out new physical understandings on the low-frequency global unsteadiness of open flows as the flapping effect occurring in separation bubbles, ${ }^{7}$ which was explained in terms of an in- teraction of modes. The regeneration of the wave packet traveling along the flow under the influence of a convective mechanism leads to a low-frequency beating.

Furthermore, Blackburn et al. ${ }^{27}$ and Marquet et al. ${ }^{28}$ studied the convective instability mechanism emerging in separated flow over a step by means of an optimization strategy involving the integration in time of the direct and adjoint linearized Navier-Stokes equations. The results obtained by such a method, called "direct optimal growth analysis" by Blackburn et al., ${ }^{27}$ confirmed the large transient growth resulting from the convective amplification of a wave packet localized in space. Such studies have thus provided a first attempt to relate the unsteadiness observed in numerical simulation and laboratory experiments in the considered flows with the noise amplifier dynamics derived from the optimal transient behavior.

It is worth to point out that all these studies present common features which rely on the non-normality of the governing linear operator, as noticed in Ref. 29. Nevertheless, none of them are dedicated to a specific investigation of the global response to an external forcing and its relationship with the onset of unsteadiness in the asymptotic regime, under the assumption of a permanent residual noise. Consequently, it seems interesting to develop a strategy based on the non-normality of temporal modes similar to the one in Refs. 20-22 in order to describe the optimal response to a harmonic forcing in the considered flow, which could result in a selective noise amplifier dynamics somewhat connected to the onset of unsteadiness observed in DNSs of separated flows by Wasistho et al., ${ }^{16}$ Pauley et al. ${ }^{30}$ and Ripley and Pauley. ${ }^{31}$

The present work, which deals with 2D globally stable flat plate separated flows, focuses on the description of the convective mechanism underlying the optimal response and investigates the effect of the high sensitivity to external forcing on the destabilizing physics in the asymptotic regime. The paper is organized as follows. In Sec. II a brief description is given of the numerical tools employed for the computation of the base flow.

In Sec. III, an analysis of the non-normality of the temporal evolution operator is provided by the computation of pseudospectra. Such an analysis is found to be able to describe the triggering of convective waves in the asymptotic regime through a pseudoresonance of the temporal modes. A specific study of the resolvent norm is carried out in order to identify the most responsive disturbance and the underlying amplification mechanism. The reduced model derived from the $2 \mathrm{D}$ temporal modes summation is then validated by performing a comparison with a linearized DNS. Later, an investigation is carried out on the effects of the increase in the adverse pressure gradient and/or the Reynolds number on the resolvent norm as well as on the spatial distribution of the associated most responsive disturbance. Such results are able to bring new elements on the influence of the shear layer on the instability mechanism.

Finally, nonlinear numerical simulations are performed initialized by the considered flow perturbed randomly at the inlet points, in order to find a connection between the selective noise amplifier behavior derived from the optimal re- 


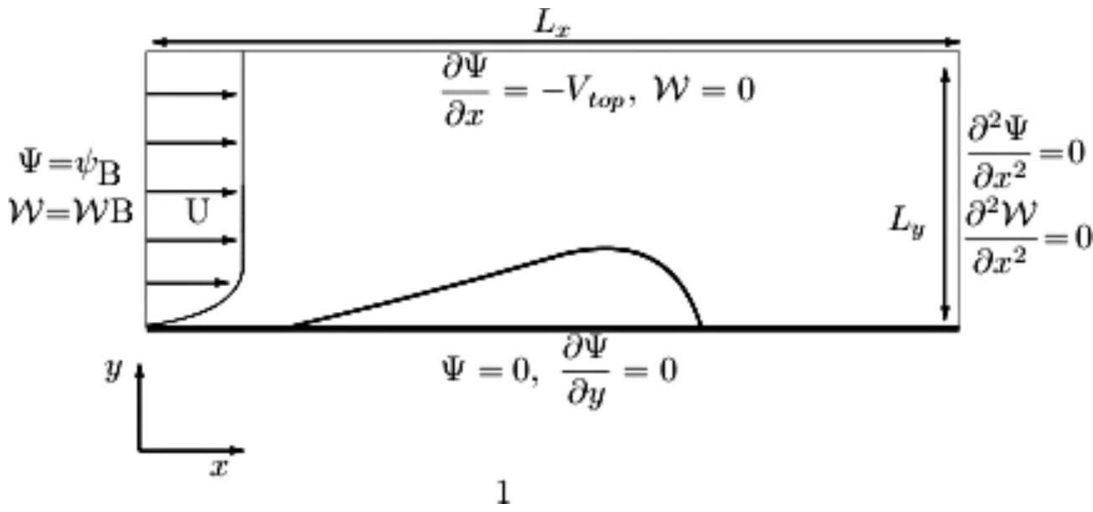

FIG. 1. Computational box and boundary conditions associated with the base flow. sponse to harmonic forcing and the onset of unsteadiness observed in separated flows. Therefore, a possible scenario is proposed related to the onset of unsteadiness in a flat plate separated flow, which is supported by the results of the present analysis and by a comparison with literature.

\section{BASIC STATES}

\section{A. Some generalities}

The incompressible 2D Navier-Stokes equations are written in the vorticity-stream function $(\mathcal{W}, \Psi)$ formulation,

$$
\begin{aligned}
& \frac{\partial \mathcal{W}}{\partial t}+U \frac{\partial \mathcal{W}}{\partial x}+V \frac{\partial \mathcal{W}}{\partial y}=\frac{1}{\operatorname{Re}}\left(\frac{\partial^{2} \mathcal{W}}{\partial x^{2}}+\frac{\partial^{2} \mathcal{W}}{\partial y^{2}}\right), \\
& \left(\frac{\partial^{2} \Psi}{\partial x^{2}}+\frac{\partial^{2} \Psi}{\partial y^{2}}\right)=\mathcal{W},
\end{aligned}
$$

where Re represents the Reynolds number and $(U, V)$ the velocity fields as $U=\partial \Psi / \partial y$ and $V=-\partial \Psi / \partial x$.

In order to induce a well defined separated zone, a specific suction profile at the upper boundary points is prescribed. A Blasius profile at the inflow, referenced as $\mathcal{W}_{B}, \Psi_{B}$ outflow and wall boundary conditions complete system (1) as depicted in Fig. 1.

\section{B. Numerical method}

The time discretization of Eq. (1) is performed using a second order Adams-Bashforth/backward-differentiation scheme based on an implicit treatment of the viscous terms and an explicit treatment of the advection terms,

$$
\begin{aligned}
& \frac{1}{\Delta t}\left(\frac{3}{2} \mathcal{W}^{n+1}-2 \mathcal{W}^{n}+\frac{1}{2} \mathcal{W}^{n-1}\right) \\
& \quad=2 \mathcal{N}\left(\mathcal{W}^{n}\right)-\mathcal{N}\left(\mathcal{W}^{n-1}\right)+\mathcal{L}\left(\mathcal{W}^{n+1}\right), \\
& \text { where } \mathcal{L}=\frac{1}{\operatorname{Re}}\left(\frac{\partial^{2}}{\partial x^{2}}+\frac{\partial^{2}}{\partial y^{2}}\right) \text { and } \mathcal{N}=-U \frac{\partial}{\partial x}-V \frac{\partial}{\partial y},
\end{aligned}
$$

where $\bullet^{n}$ is relative to the time step.

The vorticity as well as the stream function are discretized using a spectral collocation method based on Chebyshev polynomials in the wall normal direction and a second order finite difference scheme in the streamwise direction. The most difficult task in the discretization of the vorticity- stream function formulation is the definition of the vorticity at the wall where no explicit boundary condition is known. In order to provide an accurate implementation of the no-slip condition, an influence matrix method is used to compute the vorticity at the wall. A similar approach was used by Daube ${ }^{32}$ in a vorticity-velocity formulation. This technique allows to verify exactly the Neumann boundary condition of the stream function. The methodology is detailed by Peyret ${ }^{33}$ which further illustrates the numerical matters.

Consequently, at each time step four linear systems have to be solved and a matrix product $\mathcal{M}^{-1} \mathcal{S}$, giving the vorticity values at the wall, is performed. The matrix $\mathcal{M}$ is the influence matrix whereas $S$ is computed from the Neumann condition applied at boundary points on the stream function, and is updated at each time step. The build of $\mathcal{M}$ as well as its inversion are realized in the preprocessing stage. The spectral discretization in the wall normal direction coupled with the influence matrix technique allows to obtain an accurate definition of the no-slip boundary condition at the wall. Finally, a direct method based on a Thomas algorithm is used to solve the implicit system as well as the Poisson equation, taking into account the block tridiagonal matrix structure.

The DNS allows to approach a steady state solution. A Newton procedure based on the library NITSOL (Ref. 34) achieves the convergence of the base flow by means of a continuation procedure.

\section{Flat plate separated flows}

Five base flows, characterized by a different suction profile and/or Reynolds number, are computed and depicted in Figs. 2(b)-2(f). The Reynolds number $\operatorname{Re}_{\delta}$ based on the displacement thickness at $x=0$ varies from 200 to 220 which is below the critical Reynolds number for convective instabilities. The dimensions of the computational box are $L_{x}=525$ and $L_{y}=25$. A $(1000 \times 100)$ point grid allows to obtain a well converged base flow. Furthermore, such a grid is regular in the streamwise direction and clustered near the wall in the normal direction by means of a classical transformation from a Gauss-Lobatto grid.

The base flows labeled from D1 to D3 are computed at a Reynolds number $\operatorname{Re}_{\delta}=200$ for the three suction profiles displayed in Fig. 2(a). The separated flows D4 and D5 are obtained by using the same upper boundary velocity distribution as D3, for the Reynolds numbers $\operatorname{Re}_{\delta}=215$ and $\operatorname{Re}_{\delta}$ 

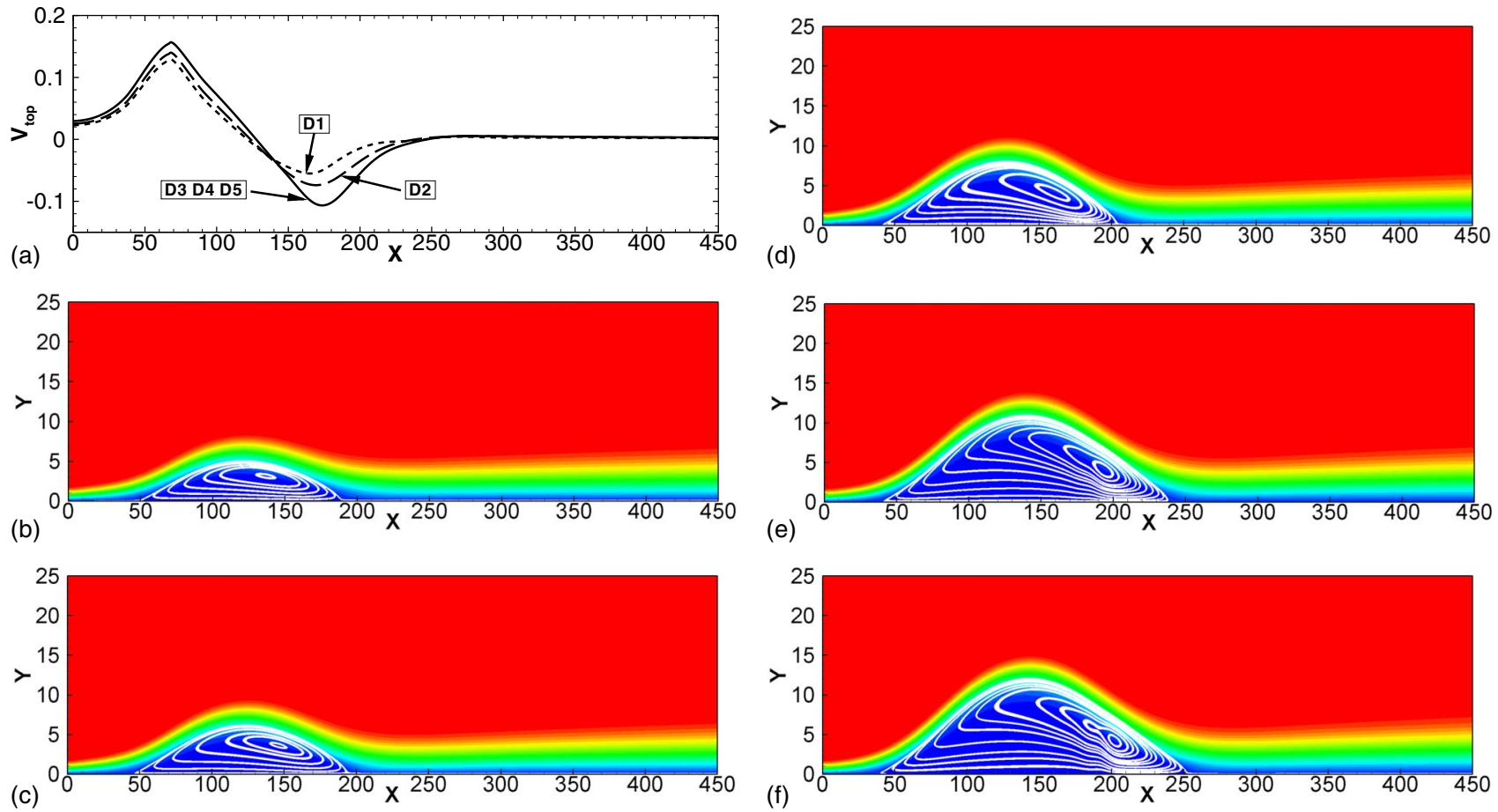

FIG. 2. (Color online) Streamwise components and streamlines of the computed separated flows. (a) Suction profiles prescribed. (b) D1. Re ${ }_{\delta}=200$. (c) D2. $\operatorname{Re}_{\delta}=200$. (d) D3. $\operatorname{Re}_{\delta}=200$. (e) D4. $\operatorname{Re}_{\delta}=215$. (f) D5. $\operatorname{Re}_{\delta}=220$.

$=220$, respectively. One may observe in Figs. 2(b)-2(f) the influence of these parameters on the resulting base flows. An increasing intensity of the suction implies a larger separated zone as well as a displacement of the center of the bubble near the reattachment point. A slight increase in the Reynolds number value causes an increase in the bubble size and of the recirculation close to the reattachment point. Moreover, under the same adverse pressure gradient, the location of the separation point is only slightly affected by the increasing of $\operatorname{Re}_{\delta}$ (Table I). The stability of such base flows is analyzed in the next sections with the aim of determining the influence of the shape of the bubble on the spatial distribution of the most responsive disturbance.

\section{CONVECTIVE INSTABILITY ANALYSIS: A SELECTIVE NOISE AMPLIFIER}

\section{A. Global linear stability: Some theory and problem definition}

In this section the base flow variables are denoted by ${ }^{\circ}$. We investigate the evolution in space and time of a small perturbation superposed to a $2 \mathrm{D}$ flat plate separated flow subject to a harmonic forcing localized in the plane $(x, y)$. A specific point in the plane $(x, y)$ will be denoted by $\mathbf{x}$ here-

TABLE I. Separation and reattachment points, referenced as $X_{s}$ and $X_{r}$, respectively, of the different base flows.

\begin{tabular}{cccccc}
\hline \hline$X$ & $\mathrm{D} 1$ & $\mathrm{D} 2$ & $\mathrm{D} 3$ & $\mathrm{D} 4$ & $\mathrm{D} 5$ \\
\hline$X_{s}$ & 49.5 & 45.8 & 40.8 & 39.8 & 39 \\
$X_{r}$ & 189 & 194 & 204 & 238 & 253 \\
\hline \hline
\end{tabular}

after. The instantaneous velocity and pressure fields $\mathbf{Q}$ are decomposed into a 2D base flow $\overline{\mathbf{Q}}={ }^{t}(\overline{\mathbf{U}}, \bar{P})$ and a $2 \mathrm{D}$ disturbance $\mathbf{q}={ }^{t}(\mathbf{u}, p)$ as follows:

$$
\mathbf{Q}=\overline{\mathbf{Q}}(\mathbf{x})+\varepsilon \mathbf{q}(\mathbf{x}, t) \text { with } \varepsilon \ll 1,
$$

with $\mathbf{u}=(u, v)$, where $u, v$ are the streamwise and wall normal components of the velocity perturbation and $p$ is the pressure perturbation. The space-time dynamics of $\mathbf{q}$ at the first order in $\varepsilon$ can thus be described by the following initial value problem:

$$
\begin{aligned}
& \mathbf{B} \frac{\partial \mathbf{q}}{\partial t}=-\mathbf{A q}+\mathcal{F}, \\
& \mathbf{q}(\mathbf{x}, t=0)=\mathbf{q}_{0},
\end{aligned}
$$

where $\mathcal{F}$ is a spatially localized forcing term and the operators $\mathbf{A}$ and $\mathbf{B}$ are defined as follows:

$$
\begin{aligned}
& \mathbf{B}=\left(\begin{array}{lll}
1 & 0 & 0 \\
0 & 1 & 0 \\
0 & 0 & 0
\end{array}\right) \text { and } \\
& \mathbf{A}=\left(\begin{array}{ccc}
\mathcal{C}_{1}-\mathcal{C}_{2}+\frac{\partial \bar{U}}{\partial x} & \frac{\partial \bar{U}}{\partial y} & \frac{\partial}{\partial x} \\
\frac{\partial \bar{V}}{\partial x} & \mathcal{C}_{1}-\mathcal{C}_{2}+\frac{\partial \bar{V}}{\partial y} & \frac{\partial}{\partial y} \\
\frac{\partial}{\partial x} & \frac{\partial}{\partial y} & 0
\end{array}\right),
\end{aligned}
$$

where $\mathcal{C}_{1}=\bar{U} \partial / \partial x+\bar{V} \partial / \partial y$ represents the effect of the advection of the perturbation by the base flow and $\mathcal{C}_{2}=\left(\partial^{2} / \partial x^{2}\right.$ 
$\left.+\partial^{2} / \partial y^{2}\right) /$ Re models the viscous diffusion effects. Appropriate boundary conditions at each edge close system (4). The base flow being convectively stable at inflow and convectively unstable at the outflow, a zero velocity perturbation, and a Robin condition, based on the approximation of the local dispersion relation, are imposed at $x=0$ and $x=L_{x}$, respectively (similar conditions are used in Refs. 20 and 21). Finally, the velocity fluctuations are set to zero at $y=0$ and $y=L_{y}$.

The operator associated with the initial value problem of the homogeneous part of Eq. (4) being independent of time, solutions are thus assumed of the form

$$
\mathbf{q}(\mathbf{x}, t)=\hat{\mathbf{q}}(\mathbf{x}) e^{-i \Omega t},
$$

where no explicit dependence in the plane $(x, y)$ is imposed and where $\Omega_{r}$ and $\Omega_{i}$ are the circular frequency and the temporal amplification rate, respectively. This ansatz allows the transformation of the homogeneous part of Eq. (4) into a large generalized eigenvalue problem,

$$
(\mathbf{A}-i \Omega \mathbf{B}) \hat{\mathbf{q}}=0,
$$

where $\hat{\mathbf{q}}(\mathbf{x})$ is the eigenfunction and $i \Omega$ is the eigenvalue. The couple $(\hat{\mathbf{q}}, \Omega)$ defines a temporal mode.

Due to the slow convergence of the spectrum, the streamwise discretization is modified, and a Chebyshev/ Chebyshev spectral collocation is used to solve system (7) (the treatment of spurious modes is illustrated in Refs. 21, 33 , and 35). For that purpose, a spectral interpolation procedure allows to transfer the base flow from the DNS grid to the stability grid. Finally, the most significative part of the spectrum is given by a shift and invert Arnoldi algorithm from the ARPACK library. ${ }^{36}$

A $(270 \times 55)$ grid discretizing a domain of lengths $L_{x}$ $=450, L_{y}=25$ for D1, D2, D3 and $L_{x}=450, L_{y}=30$ for D4, D5, and a 2000 modes Krylov subspace are assumed throughout the rest of the paper. The lengths of the computational domains have been proven to be large enough to not influence the stability results. The previously mentioned discretization yields to large, quite converged spectra for all the base flows, which are shown in Fig. 3. One can observe that all the temporal modes are damped temporally which indicates that the considered separated flows are asymptotically globally stable. In particular, the different base flows (D1D5) span many configurations, from widely stable to marginally stable flows.

Three families of temporal modes, referenced as F1, F2, and F3 and described briefly hereafter, could be recovered in the spectra. The least damped modes denoted by F1 and represented by diamonds in the top frame of Fig. 3 are reminiscent of classical Kelvin-Helmholtz $(\mathrm{KH})$ waves along the shear layer, and relax to Tollmien-Schlichting waves on the attached boundary layer. The eigenvectors of the typical modes labeled M1 and M2 in Fig. 3 are shown in Figs. 4(a) and 4(b). A second category of modes referenced as F2 and represented by squares in the top frame of Fig. 3 are characterized by a spatial distribution reaching the outflow boundary, as depicted in Figs. 4(c) and 4(d), which show the modes labeled M3 and M4 in Fig. 3. Finally, a wide range of highly damped modes are classified into F3. This last set of modes

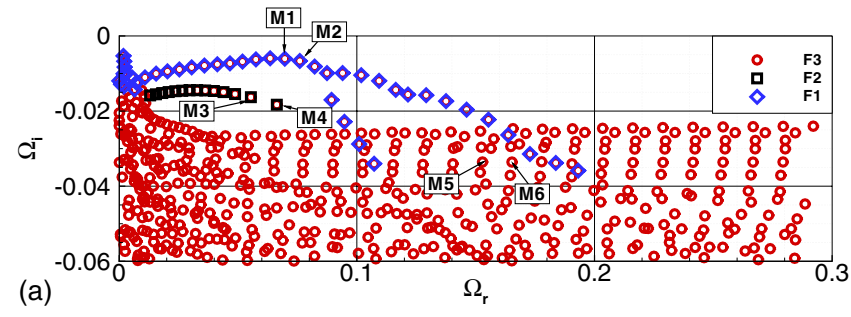

(a)

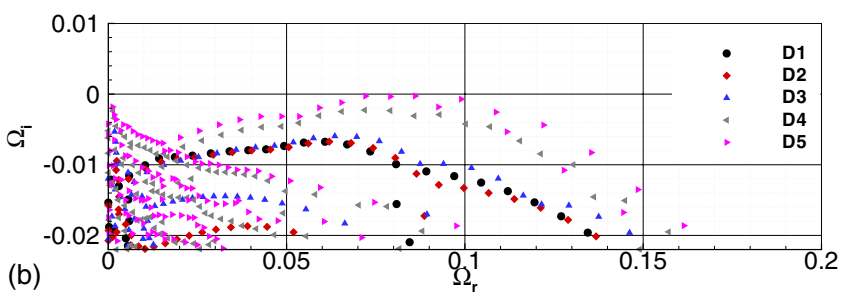

FIG. 3. (Color online) (a) Spectrum of the separated flow D3 at $\operatorname{Re}_{\delta}=200$ and $L_{x}=450$. (b) A zoom on the least damped area of the spectrum for D1-D5.

are reminiscent of the so-called continuous branch obtained by a local analysis. Indeed, a part of the energy of the corresponding modes is concentrated at high values of $y$. Furthermore, one may observe that $\mathrm{KH}$ waves are present on the shear layer as shown in Figs. 4(e) and 4(f), depicting the modes M5 and M6, respectively. Looking at Figs. 4(a)-4(f), one can notice the strong similarity in terms of spatial structure of the eigenmodes characterized by close frequencies for F1, F2, and F3. Such a property reveals the strong nonorthogonality of the eigenvectors for the considered flow, which seems to be a typical feature of open flows and, in particular, of separated flows. ${ }^{24,26}$ In Sec. III B we would investigate how the non-normality of the temporal modes could affect the linear asymptotic instability of the considered flow subject to a harmonic forcing.

\section{B. Sensitivity associated with the non-normality: Pseudoresonance}

In this section, we will investigate briefly the nonnormality features of the linearized Navier-Stokes operator and their consequences on the asymptotic behavior of the separated flows. Indeed, even if all the modes are damped temporally, the flow, being strongly separated, is subject to convective instabilities. Thus, the existence of convective waves as a response to a harmonic forcing $\mathcal{F}=\mathbf{f}(x, y) e^{-i \Omega_{f t} t}$ is investigated in a global framework. Such a phenomenon may be related to a pseudoresonance of the temporal modes due to the non-normality of the linearized Navier-Stokes operator for the considered flow. ${ }^{37}$ In order to investigate such a possibility, let us introduce the pseudospectrum of the operator,

$$
\begin{aligned}
& \lambda_{\varepsilon}=\left\{\sigma \in \mathbb{C},\|\mathbf{P}(\sigma)\| \geq \varepsilon^{-1}\right\}, \\
& \text { with } \mathbf{P}(\sigma)=(i \sigma \mathbf{B}-\mathbf{A})^{-1},
\end{aligned}
$$

which characterizes the asymptotic behavior of the evolution Eq. (4) ${ }^{37}$ In the present computations an approximation of the pseudospectrum with the Hessenberg matrix from the Arnoldi computation is used. ${ }^{21,38}$ 

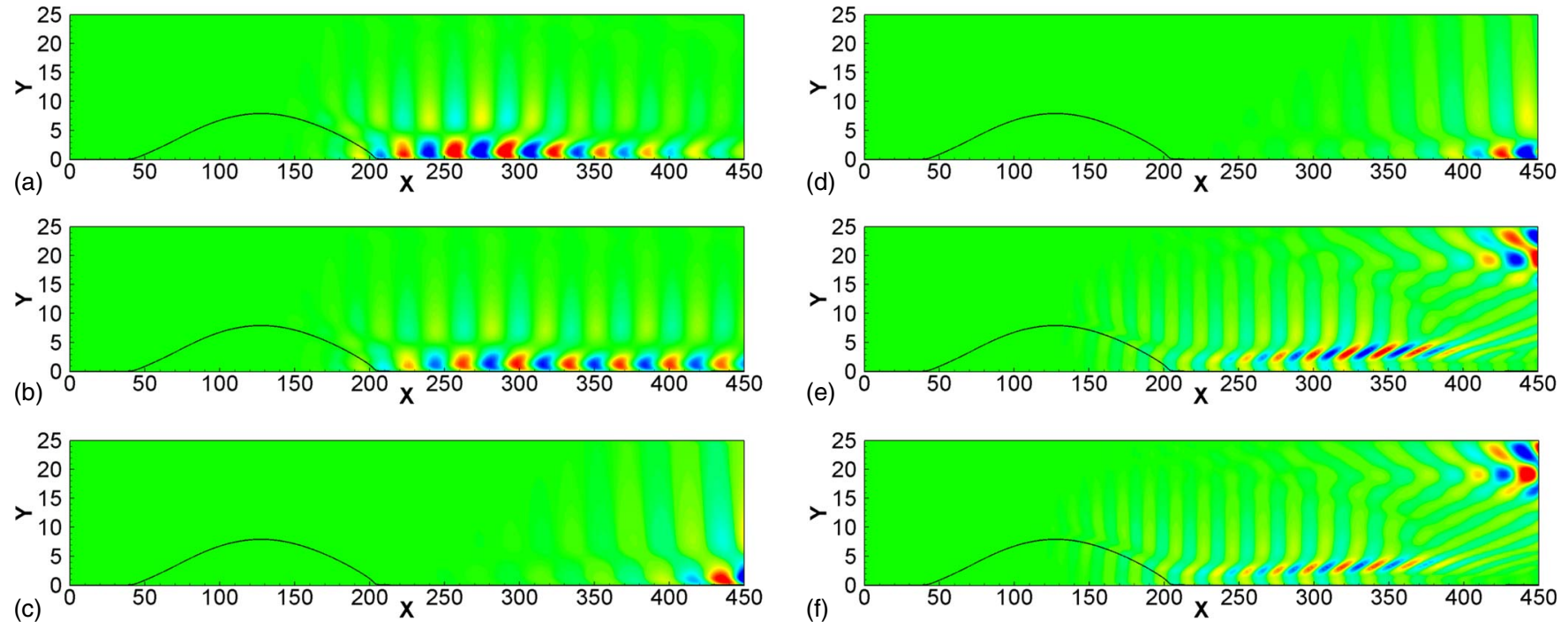

FIG. 4. (Color online) Real part of the streamwise component $\hat{u}$ of the modes labeled in Fig. 3 as M1-M6. The dividing streamline is illustrated in black. D3 is considered. (a) Mode M1. (b) Mode M2. (c) Mode M3. (d) Mode M4. (e) Mode M5. (f) Mode M6.

The base flow D1 is considered. As shown in Fig. 5, large sensitivity areas appear around each temporal mode. A pseudoresonance, resulting from the high nonorthogonality, may thus occur even far away from the considered modes. In particular, the value of the contour $-\log _{10}(\varepsilon)$ crossing the real pulsation axis represents the sensitivity of the flow to external disturbances at a given real pulsation $\Omega_{f}$; a high value of such parameter $\left(\approx 10^{-6}\right.$ for the case here considered $)$ means that the flow is able to get a large response to an external real forcing at a selected frequency $\Omega_{f}$. Furthermore, it is worth to notice that such a value does not depend on the domain size, as shown by the $\varepsilon$ levels in Fig. 5, so that one can argue that it is an intrinsic property of the separated flow. Following the arguments above, we will now investigate in detail the shape of the perturbation inducing an optimal response to a harmonic forcing and the associated sensitivity mechanism for the flow here considered.

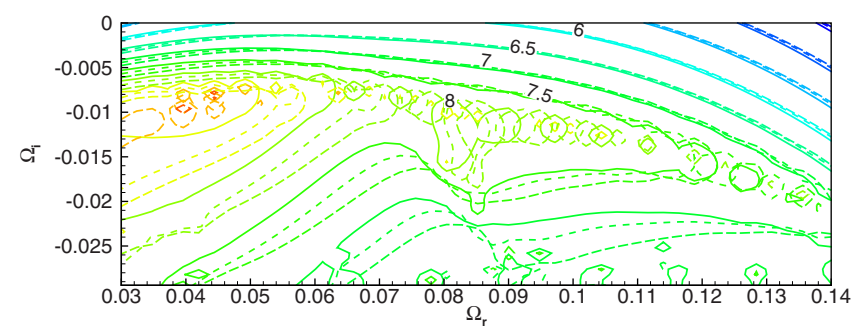

FIG. 5. (Color online) Pseudospectrum of the separated flow. The isolevels $-\log _{10}(\varepsilon)$ are shown. Three domain sizes of D1 are studied: $L 1=450(-)$, $L 2=425(--)$, and $L 3=400(--)$ discretized by $N_{x}=270,265$, and 260 grid points, respectively.

\section{Global space-time response to a localized harmonic forcing}

\section{Theoretical tools based on temporal mode expansion}

The global asymptotic response of a perturbation to a harmonic forcing $\left\{\mathcal{F}(\mathbf{x}, t)=\mathbf{f}(\mathbf{x}) e^{-i \Omega_{f}}, \Omega_{f} \in \mathfrak{R}\right\}$ can be formulated as a summation of temporal modes as follows: 22,37

$$
\mathbf{q}(\mathbf{x}, t)=\sum_{k} K_{k} \hat{\mathbf{q}}_{k}(\mathbf{x}) e^{-i \Omega_{k^{t}}},
$$

where each couple $\left(\hat{\mathbf{q}}_{k}, \Omega_{k}\right)$ is solution of Eq. (7) and $K_{k}$ characterize the initial components of the perturbation into the basis composed of temporal modes. The forcing term is expanded in a similar way,

$$
\mathbf{f}(\mathbf{x})=\sum_{k} f_{k} \hat{\mathbf{q}}_{k}(\mathbf{x}) .
$$

Under the assumption that all the temporal modes are damped temporally, in the asymptotic regime the flow's response to $\mathcal{F}$ reduces to

$$
\mathbf{q}(\mathbf{x}, t)=\sum_{k} \frac{i f_{k}}{\left(\Omega_{f}-\Omega_{k}\right)} \hat{\mathbf{q}}_{k}(\mathbf{x}) e^{-i \Omega_{f} t}
$$

We introduce now the quantity $R\left(\Omega_{f}\right)$ which characterizes the maximum response of the separated flow to a forcing,

$$
R\left(\Omega_{f}\right)=\max _{\mathcal{F}} \frac{\|\mathbf{q}\|_{E}}{\|\mathcal{F}\|_{E}}
$$

where the energy based norm \|\|$_{E}$ is derived from the scalar product $\langle\mathbf{q}, \mathbf{q}\rangle_{E}=\int_{0}^{L_{x}} \int_{0}^{L_{y}}\left(u^{*} u+v^{*} v\right) d x d y$ with •* denoting the complex conjugate. In order to evaluate Eq. (12) we introduce the scalar product matrix $\mathbf{M}$ whose coefficients are defined by 


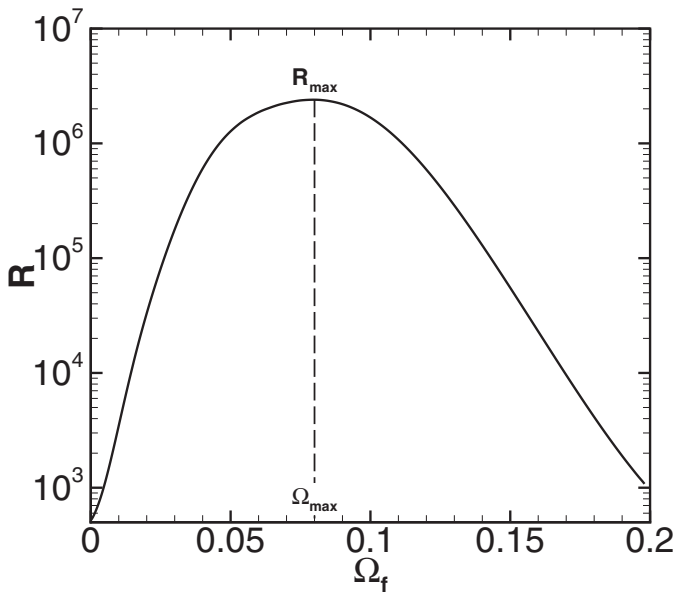

FIG. 6. The resolvent norm $R\left(\Omega_{f}\right)$ is depicted for D1. $N=1300$ modes are considered.

$$
M_{i, j}=\int_{0}^{L_{x}} \int_{0}^{L_{y}}\left(\hat{u}_{i}^{*} \hat{u}_{j}+\hat{v}_{i}^{*} \hat{v}_{j}\right) d x d y .
$$

It is thus more convenient to compute Eq. (12) as

$$
R\left(\Omega_{f}\right)=\left\|\mathbf{F D}_{f} \mathbf{F}^{-1}\right\|_{2},
$$

with $D_{f(l, p)}=\delta_{l, p}\left[i /\left(\Omega_{f}-\Omega_{l}\right)\right]$ and $\mathbf{M}=\mathbf{F}^{t} \mathbf{F}$ the Cholesky decomposition of $\mathbf{M}$.

Finally, Eq. (14) can be computed for each $\Omega_{f}$ by determining the largest singular value $s v_{1}$,

$$
R\left(\Omega_{f}\right)=s v_{1}\left(\mathbf{F D}_{f} \mathbf{F}^{-1}\right) .
$$

The expression of the most responsive disturbance is represented in the temporal mode expansion by the vector $\mathbf{K}_{\text {res }}$ which is equal to the right singular vector of $\mathbf{F} \mathbf{D}_{f} \mathbf{F}^{-1}$ associated with the largest singular value $s v_{1}$. The components of the forcing term $\mathbf{K}_{f}={ }^{t}\left(f_{1}, f_{2}, \ldots, f_{N}\right)$ leading to such a response can thus be recovered by a simple matrix product $\mathbf{K}_{f}=\mathbf{F}^{-1} \mathbf{K}_{\text {res }}$.

\section{Validation of the reduced model}

The influence of the number of modes $N$ taken into account in the optimization process is investigated. For that purpose, the base flow D1 is considered. Similar results are provided for the different base flows. The resolvent norm $R$ is plotted in Fig. 6 for $N=1300$. As it is suggested by the pseudospectrum analysis, one observes a strong amplification of the value of $R$ which reaches a maximum value for $\Omega_{f}=0.08$. In order to validate the behavior predicted by a limited number of modes, two quantities are introduced, denoted by $R_{\max }(N)$ and $\Omega_{\max }(N)$,

$$
\begin{aligned}
& R_{\text {max }}(N)=\max _{\Omega_{f}}\left[R\left(\Omega_{f}, N\right)\right], \\
& \Omega_{\text {max }}(N)=\left\{\Omega_{f} / R\left(\Omega_{f}, N\right)=R_{\text {max }}(N)\right\},
\end{aligned}
$$

which are sketched in Fig. 6. The values of such quantities in function of the number of modes used in the computation of $R\left(\Omega_{f}\right)$ are depicted in Figs. 7(a) and 7(b). One observes a good convergence of the most amplified frequency which reaches an almost constant value for $N=800$. The value of
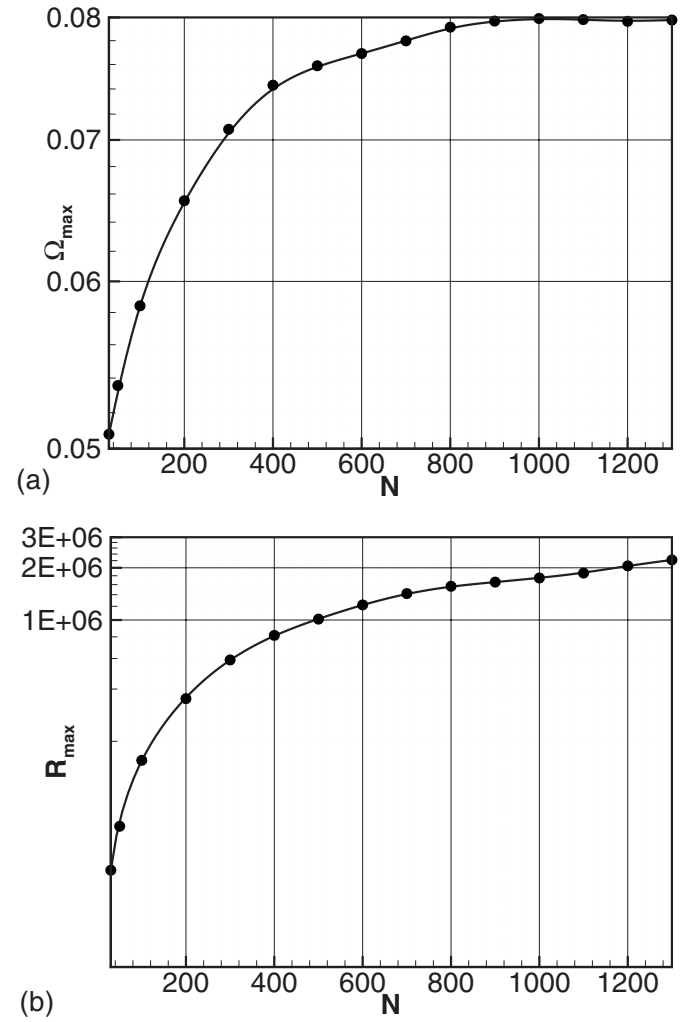

FIG. 7. Influence of the dimension of the temporal mode expansion for D1 and $L_{x}=450$. (a) Evolution of $\Omega_{\max }$ with the number of modes. (b) Evolution of $R_{\max }$ with the number of modes.

$R_{\max }$ exhibits a fast increase for moderate values of $N$ and a slow increase after $N=800$. One may expect convergence when $N \rightarrow \infty$.

Therefore, $N=1300$ modes are here considered sufficient to capture the optimal response and its associated frequency. The spatial distribution of the optimal forcing and its respective response, both represented by the variable $U_{x}(x)$ $=\int_{0}^{L_{y}} u(x, y)^{2} d y$, are shown in Figs. 8(a) and 8(b) (the quantities are normalized by $\left.\max _{x}\left[U_{x}(x)\right]\right)$. It appears that a number of modes greater than $N=800$ lead to a quite converged spatial distribution of the optimal response and forcing, whose $u$

(a)

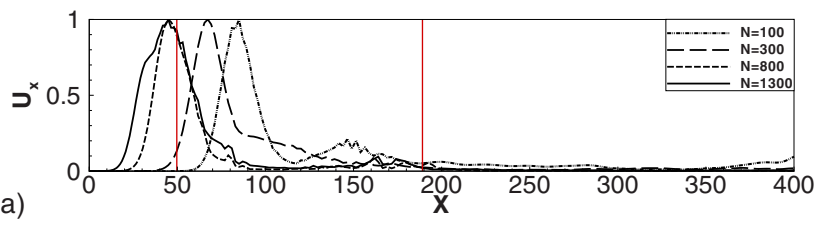

(b)

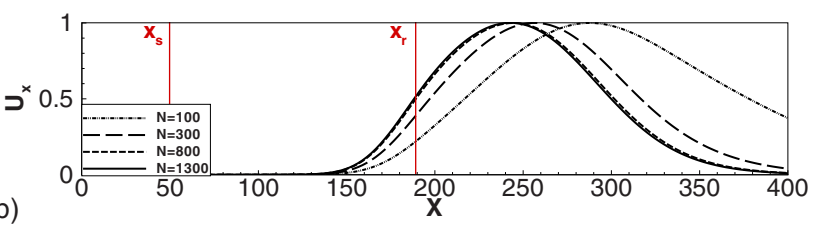

FIG. 8. (Color online) Influence of the number of modes $N$ on the spatial distribution of the optimal forcing and response for D1 and $L_{x}=450$. The vertical lines correspond to the separation point and the reattachment point denoted by $X_{s}$ and $X_{r}$ respectively. (a) Streamwise distribution of the optimal forcing represented by the variable $U_{x}(x)$. (b) Streamwise distribution of the optimal response represented by the variable $U_{x}(x)$. 

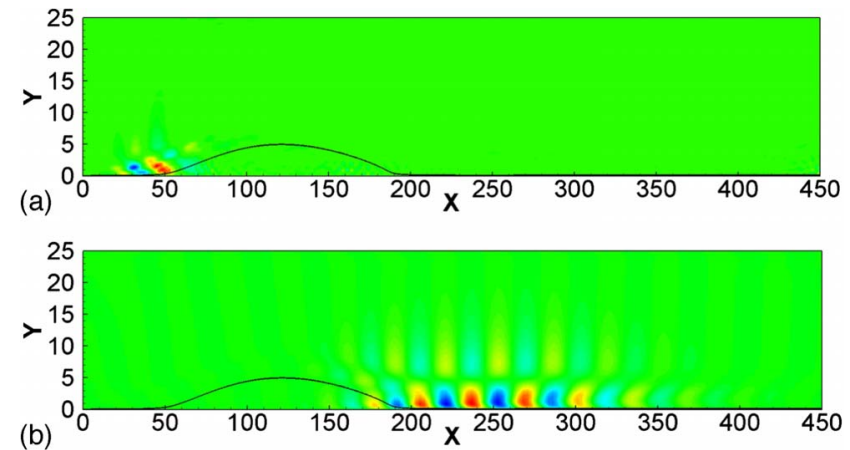

FIG. 9. (Color online) Representation of the streamwise component resulting from the optimization process. D1 is considered. (a) Streamwise component $u$ of the optimal forcing. (b) Streamwise component $u$ of the optimal response.

components are shown in Figs. 9(a) and 9(b). One can observe that for $N=1300$, the optimal forcing is localized close to the separation point of the bubble leading to a response which is amplified along the shear layer reaching a maximum after the reattachment point. Consequently, it could be argued that the optimal frequency and the corresponding response are well described by a limited number of modes despite the slow convergence of $R_{\max }$. In particular, such a slow convergence may be ascribed to the difficulty to recover the tilting of the initial perturbation upstream of the bubble by means of a reduced model based on a global mode expansion. Indeed, the action of the shear on structures tilted at $t=0$ in the direction opposed to the mean flow may lead, through an Orr mechanism, to an increase in the energy gain, as observed by Blackburn et $a .^{27}$ and Marquet et al. ${ }^{28}$ by means of a direct-adjoint optimal growth strategy. On the other hand, such a mechanism is not perfectly observed in the works of Åkervik et al. ${ }^{24}$ and Ehrenstein and Gallaire, ${ }^{26}$ where a global mode expansion strategy is used. Furthermore, in the work of Åkervik et al. ${ }^{22}$ on a flat plate boundary layer, it is demonstrated that the Orr mechanism does not affect the value of the optimal response frequency but only the value of the maximum energy gain.

Finally, a validation of the previously discussed results is carried out by means of a perturbative linearized version of the DNS code described in the first part. The equations are written as follows:

$$
\begin{aligned}
& \frac{\partial \varpi}{\partial t}+\overline{\mathbf{U}} \cdot \nabla \varpi+\mathbf{u} \cdot \nabla \overline{\mathcal{W}}=\frac{1}{\operatorname{Re}} \nabla^{2} \varpi+\mathcal{F}, \\
& \nabla^{2} \psi=\varpi,
\end{aligned}
$$

where $\mathcal{F}$ represents the forcing term, and $\varpi$ and $\psi$ the vorticity and the stream function perturbation, respectively. The nullity of the velocity fluctuations is imposed all over the boundaries of the computational domain except at the outflow where the second derivatives of $\varpi$ and $\psi$ along $x$ are set to zero. A fringe region is implemented in order to damp the perturbation at the outlet.

We initialize at zero the perturbation fields and force with two circular frequencies $\Omega_{f}=0.08$ and $\Omega_{f}=0.13$. The associated forcing field is deduced from the optimization
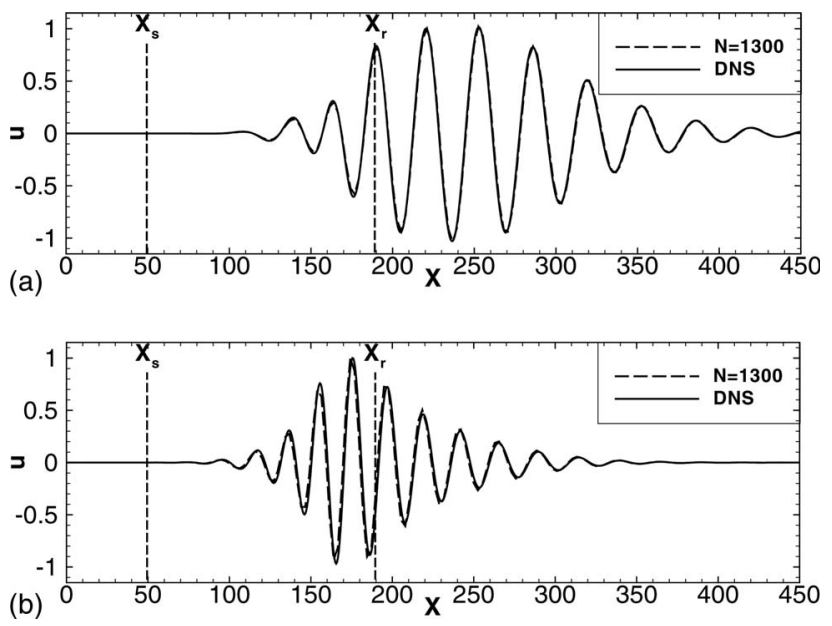

FIG. 10. Plot of the value of $u(x, 8)$ obtained by linearized DNS and temporal mode expansion with $N=1300$ for the base flow D1. (a) $\Omega_{f}=0.08$. (b) $\Omega_{f}=0.13$.

process. The grid and domain lengths discussed in Sec. II C are used. After the transient, we compare the instantaneous perturbation with the one resulting from the temporal mode expansion with $N=1300$. Figures 10 (a) and 10(b) show that the linearized simulation results are in a very good agreement with the ones of the linear stability theory based on summation (9). Such a computation is a good validation of the temporal mode expansion which has demonstrated to be an appropriate reduced model for the description of the optimal response to external forcing in the asymptotic regime. Being confident about the results derived from the present summation, we further explore the physical destabilizing mechanism associated with the optimal response.

\section{Physical mechanism associated with the optimal response}

In Fig. 11 we plot the vector perturbation fields $(u, v)$ for $\Omega_{\max }$ in the asymptotic regime for the base flow D3. One may observe that the action of the perturbation on the base flow originates a series of counter-rotating vortices along the shear layer and the decelerated zone. A visualization of the instantaneous vorticity field, where $\varepsilon$ is fixed arbitrarily to $10 \%$ of the maximum value of $\bar{U}$, represents the resulting response in Fig. 12. The destabilizing mechanism associated with the most responsive disturbance described above leads to the formation of rolling vortices amplified along the shear layer which are advected in the attached boundary layer and die away. Such process seems to be a specific feature under-

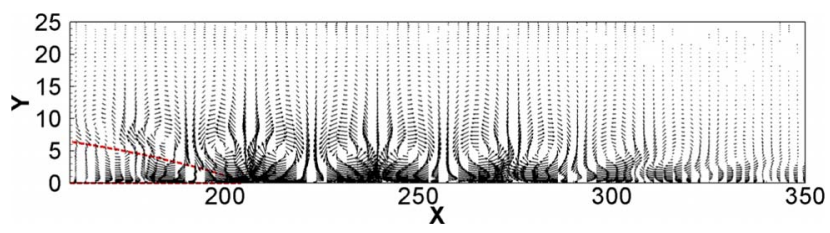

FIG. 11. (Color online) Illustration of the perturbation vectors $(u, v)$ obtained for D3 and the forcing frequency $\Omega_{\max }$ in the asymptotic regime. The dividing streamline is represented by the dashed line. 


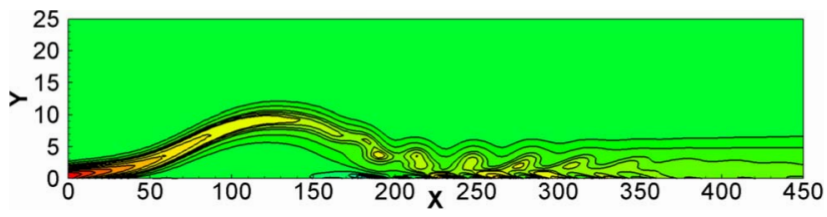

FIG. 12. (Color online) Instantaneous vorticity for D3 and $\Omega_{\max }$ in the asymptotic regime.

lying the optimal behavior of separated flows. We may for instance refer to the counter-rotating structures observed by Blackburn et $a .^{27}$ in a flow over a backward facing step resulting from the optimal amplification of a localized wave packet.

In order to determine the effects of the size of the recirculation area on the values of $R\left(\Omega_{f}\right)$, computations are performed for the different base flows discussed in Sec. II C. The response curves are shown in Fig. 13.

One can observe the strong increase of the optimal response value $R_{\max }$ with the size of the recirculation zone which reaches a very high maximum energy gain of order $\approx 10^{9}$ for D5. In particular, the most responsive frequency range is found to reduce when the Reynolds number and/or the pressure gradient at the upper boundary increase, leading to a more defined peak around $\Omega_{\max }$.

Furthermore, Figs. 14-16 show that the spatial support associated with the most responsive disturbance is spreading along the separated zone with the increase in the recirculation area. In particular, this last one is centered around the reattachment point for D4. This correlation suggests that an inviscid-type $\mathrm{KH}$ mechanism, similar to the one observed in separated flows by Lin and Pauley, ${ }^{39}$ for instance, is the cause of the increase in the optimal response energy. As described by the latter, this last one results in the roll-up of the shear layer.

\section{Relation between the global optimal response and the onset of unsteadiness}

Our analysis has assessed that flat plate separated flows may exhibit a large response to a harmonic forcing for a range of frequencies at moderate Reynolds number. The bubble may act as a selective amplifier of frequencies due to

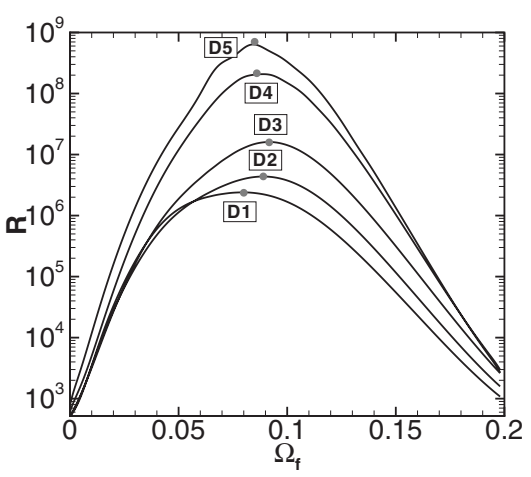

FIG. 13. Evolution of $R\left(\Omega_{f}\right)$ for the different separated flows discussed in Sec. II C. $R_{\max }$ are sketched in black points.

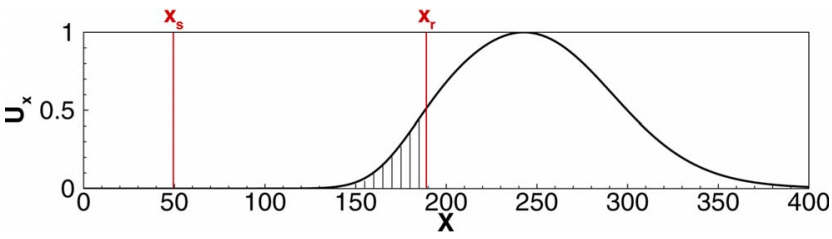

FIG. 14. (Color online) Streamwise distribution of the optimal response for the base flow D1.

the strong convectively unstable character of the flow. Furthermore, a typical feature of such configuration is the onset of self-sustained oscillations characterized by the triggering of shedded vortices (see Refs. 16, 30, and 31 in DNS and Ref. 40 in experiments). Moreover, such flows are subject to a certain level of noise in experiments as well as in numerical simulations due to the discretization errors. Therefore, we propose to investigate the connection between the emergence of coherent structures in the considered configuration subject to noise and the amplifier dynamics related to the optimal response to forcing, which leads to the generation of counterrotating structures, as previously discussed. For that purpose, the full 2D Navier-Stokes equations by superposing a random noise with small amplitude $\left(10^{-6}\right)$ to the base flow inlet vorticity are computed. Simulations are carried out for the base flow D5. Figure 17 shows a time series and a Fourier analysis of the vorticity component at the wall extracted at two different locations, the reattachment points $x=253$ and $x=282$; after that the simulation has reached a statistically stationary state. Although a white noise perturbation is imposed at the inflow, the Fourier spectra shown in Figs. 17(b) and $17(\mathrm{c})$ provide a narrow banded response whose peak is localized around $\Omega=0.085$. This behavior is in agreement with the optimal response analysis realized in Sec. III C 1. In particular, the peak and the range of excited pulsations are close to the predictions of the theoretical analysis based on a temporal modes summation. Similar results are observed with D1-D4.

Thus, we can assume that the amplification mechanism provided by the optimal response analysis is able to describe a possible scenario for the onset of unsteadiness in the considered separated flow. Under a sufficiently large pressure gradient and/or Reynolds number, even a 2D globally stable separated flow is able to select by means of sensitivity an optimal range of frequencies out of the existing numerical noise, generating optimal-like counter-rotating structures which can trigger a self-excited vortex shedding.

This selective noise amplifier mechanism can be compared to the unsteadiness taking the form of $\mathrm{KH}$-like shedded

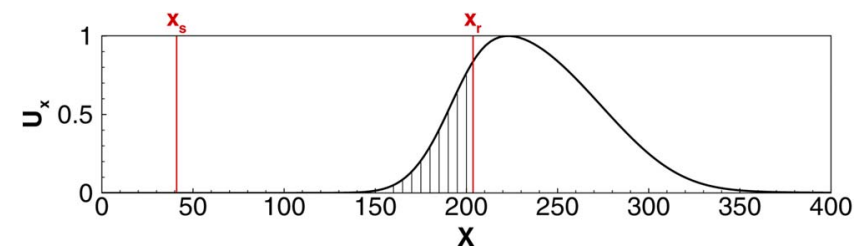

FIG. 15. (Color online) Streamwise distribution of the optimal response for the base flow D3. 


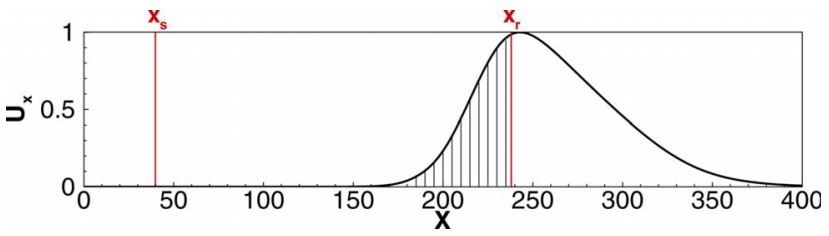

FIG. 16. (Color online) Streamwise distribution of the optimal response for the base flow D4.

vortices observed in the asymptotic regime in numerical simulations of similar configurations by Wasistho et al., ${ }^{16}$ Pauley et al..$^{30}$ and Ripley and Pauley. ${ }^{31}$

Indeed, such authors observed that under a sufficiently large pressure gradient, a strongly unsteady behavior taking the form of self-excited vortex shedding occurs even at low Reynolds number $\left(\operatorname{Re}_{\delta}=330\right.$ for the first one and from 400 to 800 for the last one). A natural generation of convective waves from the existing numerical noise is thus suggested by Ref. 16 as an explanation.

Focusing on the relation between optimal response and the vortex shedding observed in previous investigations, we compute a typical parameter characterizing the unsteadiness behavior such as the shedding frequency nondimensionalized by the boundary layer momentum thickness $\theta_{s}$ and the local free stream velocity $U_{e}$ at the separation point with no applied gradient pressure, through Strouhal number,

$$
S_{t \theta}=f \frac{\theta_{s}}{U_{e}},
$$

where $f$ denotes the shedding frequency. According to Pauley et al. ${ }^{30}$ Ripley and Pauley ${ }^{31}$ and Pauley ${ }^{41}$ for flat plate separated flows or Lin and Pauley ${ }^{39}$ in an airfoil configuration, a typical Strouhal number associated with unsteadiness varies in the range from 0.0055 to 0.008 . It is worth to notice that such a value is not fixed constant due to the dependence of
TABLE II. Values of $\theta_{s}, \Omega_{\max }$, and $S_{t \theta}$ at $X_{s}$ for the five separated flows.

\begin{tabular}{llll}
\hline \hline & $\theta_{s}$ & $\Omega_{\max }$ & $S_{t \theta}$ \\
\hline D1 & 0.508 & 0.08 & 0.00647 \\
D2 & 0.500 & 0.089 & 0.00708 \\
D3 & 0.489 & 0.092 & 0.00716 \\
D4 & 0.480 & 0.088 & 0.00673 \\
D5 & 0.477 & 0.085 & 0.00645 \\
\hline \hline
\end{tabular}

the shedding frequency to the pressure distribution along the flat plate. ${ }^{31}$ Therefore, the shedding frequency is slightly geometry dependent.

The Strouhal numbers associated with the frequency leading to an optimal response are computed and summarized in Table II. Such values are found to be consistent with the ones obtained by the authors previously mentioned.

In order to verify that the optimal response is able to trigger unsteadiness in the nonlinear regime, a larger white noise amplitude is superposed at inflow points in a DNS. The base flow D3 is investigated, and the amplitude is fixed to $10^{-5}$. After the transient, the nonlinear saturation occurs. Figure 18 shows an instantaneous vorticity field showing the vortex shedding behavior resulting from the selective noise amplifier mechanism at $t=2000$. It is worth to notice the strong similarity between the resulting $2 \mathrm{D}$ structures and the computations of previous investigations by Refs. 16, 30, and 31.

Such results support the hypothesis that flat plate separated flows could act as a selective noise amplifier, whose selected frequencies could be recovered by an optimal response analysis, and whose amplificating response leads to the onset of a self-excited vortex shedding phenomenon.

Nevertheless, as it is detailed in Sec. I of the present manuscript and recently discussed by Marquet et al., ${ }^{28}$ a
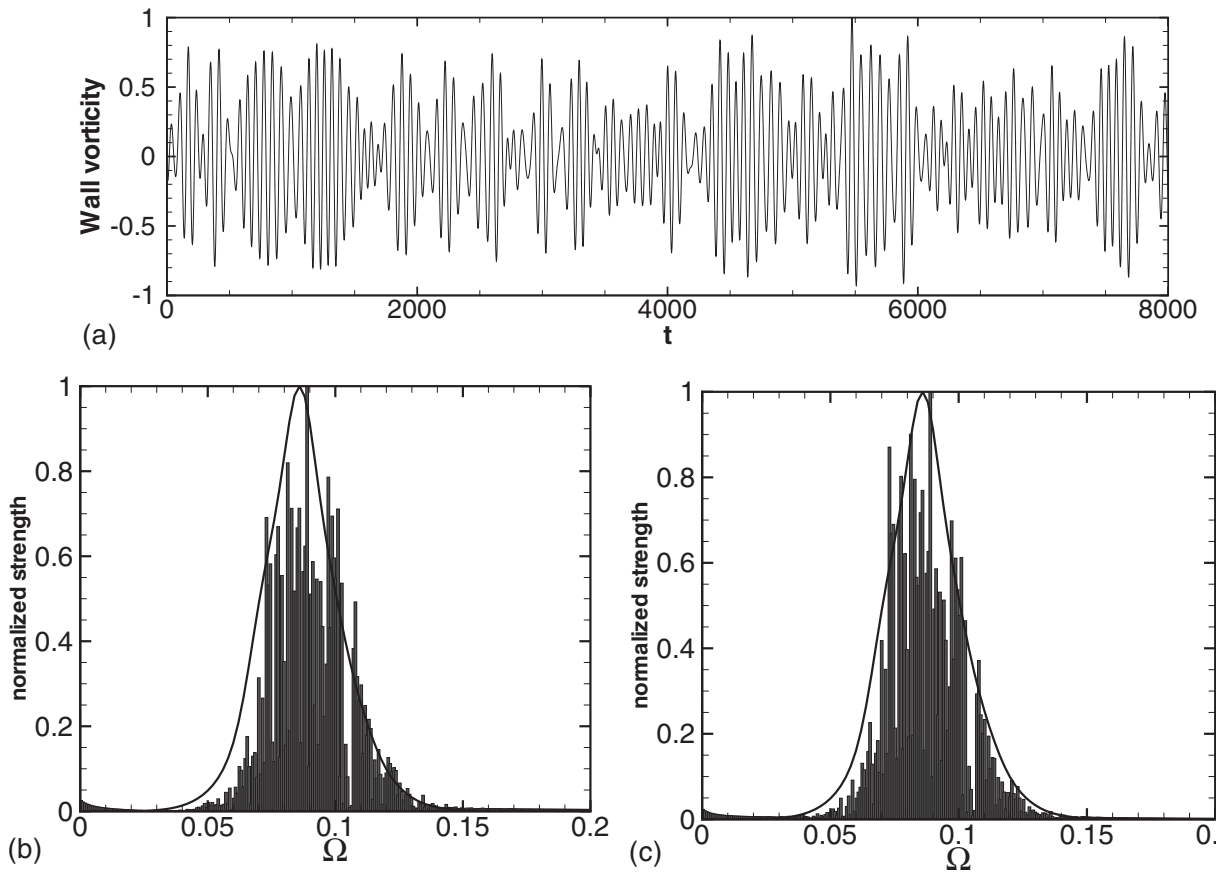

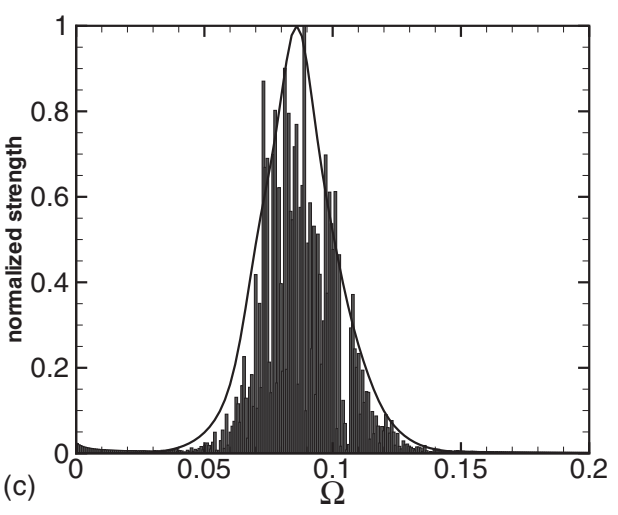

FIG. 17. Vorticity histories at the wall taken at two different positions where the inflow condition is continually perturbed with a low-amplitude random noise. The normalized optimal response to a localized harmonic forcing is depicted in black line. D5 is considered. (a) Time series of the wall vorticity at the reattachment point $x=253$ extracted from the DNS. (b) Normalized pulsation distribution from Fourier analysis of the wall vorticity history at the reattachment point $x=253$. (c) Normalized pulsation distribution from Fourier analysis of the wall vorticity history after the reattachment point $x=282$. 


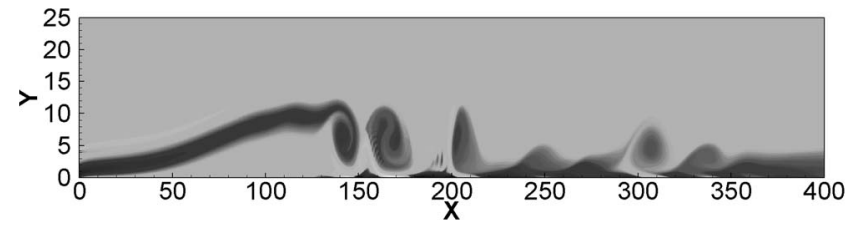

FIG. 18. Instantaneous vorticity field from DNS subject to a random white noise at the inflow. The amplitude is fixed to $10^{-5}$.

resonator dynamics associated with a three-dimensional global stationary unstable mode may occur in separated flows as it is observed in a similar configuration by Theofilis et al. ${ }^{13} \mathrm{It}$ may be supposed that such three-dimensional mechanism could appear in the present configuration. Therefore, one can argue that such resonator dynamics could dominate the asymptotic space-time dynamics of the considered flow and invalidate the present analysis. However, Gallaire et al., ${ }^{15}$ Marquet et al., ${ }^{28}$ and Blackburn et al. ${ }^{27}$ established that such three-dimensional global mode is amplified with a weak temporal growth rate. Furthermore, the analysis of the influence of random noise perturbation superposed at the inflow in a similar configuration by Pauley ${ }^{42}$ illustrates that the vortex shedding frequency observed in the $2 \mathrm{D}$ computation is not altered by the influence of a three dimensionality of the flow. Thus, it may be suggested that the selective noise amplifier associated with the optimal response yields a convincing scenario for the onset of unsteadiness in flat plate separated flows excited by a permanent external noise.

Consequently, the global analysis based on temporal mode expansion seems to be efficient in modeling the asymptotic behavior of open flows which are found to be strongly sensitive to external forcing, opening new possibili- ties of control strategy aiming at minimize the quantity $R\left(\Omega_{f}\right)$ by reducing the non-normality of the operator. ${ }^{43}$

\section{CONCLUSION}

Recently, global linear stability analyses have yielded a rich picture of the instability features of separated flows. In particular, the concept of non-normality in a global framework has explained some large transient phenomena occurring even at low Reynolds number. Therefore, as argued by Marquet et al. ${ }^{28}$ and Blackburn et al., ${ }^{27}$ the amplifier dynamics predicted by an optimal growth analysis yields a possible scenario explaining the onset of unsteadiness in flows over a backward facing step under the influence of a localized disturbance. Nevertheless, it seems interesting to adopt a different point of view connecting the triggering of unsteadiness in the asymptotic regime and the selective noise amplifier behavior of a separated flow. For that purpose, in this work we have studied the global linear response to a localized harmonic forcing leading to a maximum kinetic energy gain in the asymptotic regime, as well as the associated amplification mechanism and its connection with the onset of unsteadiness in flat plate separated flows.

The proposed global linear stability approach is found to be able to identify the instability mechanism related to the linear response to a harmonic forcing introduced into a laminar flat plate separated flow. In particular, the temporal mode expansion of the perturbation is able to describe the linear space-time dynamics of a 2D perturbation, outlining a destabilizing mechanism involving the shear layer. Indeed, the most responsive disturbance takes the form of $\mathrm{KH}$-like vortices, which roll up and amplify themselves along the shear layer, until being advected and die away in the attached

TABLE III. $V_{\text {top }}(x)$.

\begin{tabular}{|c|c|c|c|c|c|c|c|c|c|c|}
\hline$x$ & 0 & 10 & 20 & 30 & 40 & 50 & 60 & 70 & 80 & 90 \\
\hline D1 & 0.0227 & 0.0249 & 0.0308 & 0.0418 & 0.0614 & 0.0900 & 0.1155 & 0.1241 & 0.0097 & 0.0068 \\
\hline $\mathrm{D} 2$ & 0.0253 & 0.0277 & 0.0343 & 0.0467 & 0.0688 & 0.0983 & 0.1254 & 0.1352 & 0.1083 & 0.0789 \\
\hline \multirow[t]{2}{*}{ D3 } & 0.0295 & 0.0323 & 0.0401 & 0.0544 & 0.0780 & 0.1110 & 0.1406 & 0.1523 & 0.1266 & 0.0974 \\
\hline & 100 & 110 & 120 & 130 & 140 & 150 & 160 & 170 & 180 & 190 \\
\hline D1 & 0.0445 & 0.0233 & 0.0029 & -0.0164 & -0.0329 & -0.0448 & -0.0532 & -0.0529 & -0.0411 & -0.0263 \\
\hline $\mathrm{D} 2$ & 0.0547 & 0.0315 & 0.0084 & -0.0142 & -0.0357 & -0.0546 & -0.0685 & -0.0734 & -0.0670 & -0.0523 \\
\hline \multirow[t]{2}{*}{ D3 } & 0.0722 & 0.0470 & 0.0206 & -0.0066 & -0.0335 & -0.0597 & -0.0862 & -0.1041 & -0.1014 & -0.0813 \\
\hline & 200 & 210 & 220 & 230 & 240 & 250 & 260 & 270 & 280 & 290 \\
\hline D1 & -0.0149 & -0.0077 & -0.0043 & -0.0030 & -0.0013 & 0.0014 & 0.0036 & 0.0042 & 0.0038 & 0.0032 \\
\hline $\mathrm{D} 2$ & -0.0355 & -0.0212 & -0.0108 & -0.0039 & 0.0003 & 0.0028 & 0.0041 & 0.0048 & 0.0050 & 0.0050 \\
\hline \multirow[t]{2}{*}{ D3 } & -0.0557 & -0.0337 & -0.0187 & -0.0096 & -0.0034 & -0.0012 & 0.0041 & 0.0053 & 0.0054 & 0.0051 \\
\hline & 300 & 310 & 320 & 330 & 340 & 350 & 360 & 370 & 380 & 390 \\
\hline D1 & 0.0028 & 0.0027 & 0.0027 & 0.0027 & 0.0026 & 0.0025 & 0.0024 & 0.0024 & 0.0023 & 0.0023 \\
\hline $\mathrm{D} 2$ & 0.0049 & 0.0047 & 0.0045 & 0.0042 & 0.0040 & 0.0038 & 0.0037 & 0.0035 & 0.0034 & 0.0033 \\
\hline D3 & 0.0048 & 0.0047 & 0.0046 & 0.0045 & 0.0043 & 0.0041 & 0.0039 & 0.0037 & 0.0036 & 0.0035 \\
\hline
\end{tabular}


boundary layer. The pseudoresonance of the temporal modes due to the non-normality of the temporal evolution operator of the linearized Navier-Stokes equations explains this behavior. Moreover, an analysis of the evolution of the response, when the Reynolds number and/or the gradient pressure are increased, clarifies the strong influence of the shear layer on the maximum response.

Such elements suggest that flat plate separated flows may act as a strong selective noise amplifier. In order to find out if a connection exist between the global optimal response of the flow and the unsteadiness observed in experiments and DNS, a DNS is carried out in which the base flow is continuously perturbed at inlet points with a random noise. The DNS results show that the selected frequencies recovered by Fourier transform in the asymptotic regime are in agreement with the amplified frequencies derived from the optimal response analysis. The most amplified frequency is then compared to the shedding frequencies measured by Pauley et al. ${ }^{30}$ Ripley and Pauley, ${ }^{31}$ Lin and Pauley, ${ }^{39}$ and Wasistho et al. ${ }^{16}$ The Strouhal number recovered by the authors previously mentioned is found consistent with the most amplified frequency of the global optimal response.

Finally, the present analysis should be completed by taking into account the influence of three-dimensional perturbations. The emergence of a resonator dynamics associated with a stationary unstable global mode is expected. ${ }^{13}$ Therefore, the asymptotic dynamics of such flow is still a challenging problem where the amplifier and the resonator dynamics may compete.

\section{ACKNOWLEDGMENTS}

The authors would like to thank Ulrich Rist for welcoming Frédéric Alizard into the IAG and for his financial support. We would like to acknowledge the anonymous referees for enlightening discussions, their comments, and advice. Computing time was provided by "Institut du Développement et des Ressources en Informatique Scientifique (IDRIS)-CNRS."

\section{APPENDIX A: SUCTION VELOCITY PROFILES: $\boldsymbol{V}_{\text {top }}(\boldsymbol{x})$}

Table III defines the values taken by $V_{\text {top }}$ along some streamwise position $x$.

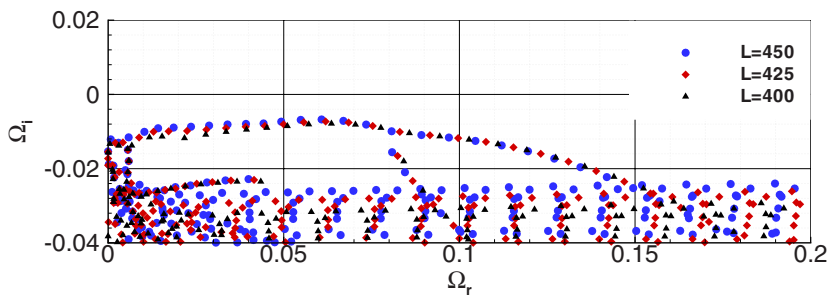

FIG. 19. (Color online) Influence of the computational box on the spectrum. D1 is considered.

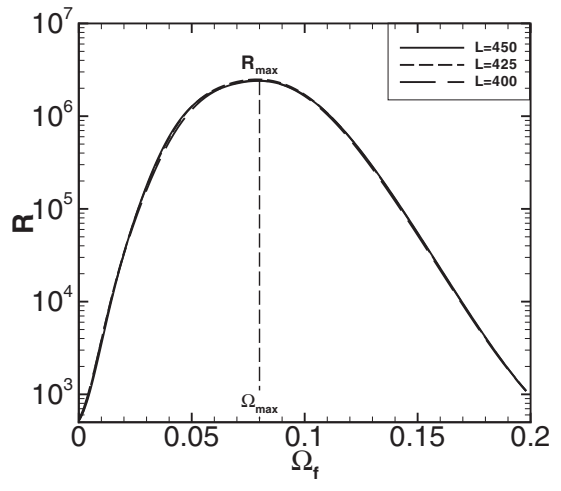

FIG. 20. The resolvent norm $R\left(\Omega_{f}\right)$ is depicted for D1 where 1300 modes are considered. Three computational boxes are studied referenced by $L_{x}$ $=450, L_{x}=425$, and $L_{x}=400$ associated with $N_{x}=270, N_{x}=265$, and $N_{x}$ $=260$, respectively.

\section{APPENDIX B: COMPUTATIONAL BOX DEPENDENCY}

This appendix is devoted to the analysis of the dependency of the stability results on the computational box. The case D1 is considered. From Figs. 19 and 20, one observes that despite the spectrum is influenced by the domain size, the resulting resolvent norm is unchanged. The global values are thus independent of the computational box.

${ }^{1}$ B. M. Jones, "Stalling," J. R. Aeronaut. Soc. 38, 753 (1934).

${ }^{2}$ M. Gaster, "The structure and behaviour of laminar separation bubbles," Ministry of Technology, Aeronautical Research Council Technical Report No. 3595, 1969.

${ }^{3}$ W. R. Briley, "A numerical study of laminar separation bubbles using the Navier-Stokes equations," J. Fluid Mech. 47, 713 (1971).

${ }^{4}$ V. Sychev, A. I. Ruban, V. Sychev, and G. L. Korolev, Asymptotic Theory of Separated Flows (Springer, New York, 2005).

${ }^{5} \mathrm{U}$. Rist and U. Maucher, "Direct numerical simulation of 2-d and 3-d instability waves in a laminar separation bubble," AGARD Conf. Proc. 551, 361 (1994).

${ }^{6} \mathrm{U}$. Rist and U. Maucher, "Investigations of time-growing instabilities in laminar separation bubbles," Eur. J. Mech. B/Fluids 21, 495 (2002).

${ }^{7}$ A. V. Dovgal, V. V. Kozlov, and A. Michalke, "Laminar boundary layer separation: Instability and associated phenomena," Prog. Aerosp. Sci. 30, 61 (1994).

${ }^{8}$ B. F. Armaly, F. Durst, J. C. F. Pereira, and B. Schönung, "Experimental and theoretical investigation of backward-facing step flow," J. Fluid Mech. 127, 473 (1983)

${ }^{9}$ L. Kaiktsis, G. Karniadakis, and A. Orszag, "Unsteadiness and convective instabilities in two-dimensional flow over a backward-facing step," J. Fluid Mech. 321, 157 (1996).

${ }^{10} \mathrm{P}$. Huerre and P. A. Monkewitz, "Absolute and convective instabilities in free shear layers," J. Fluid Mech. 159, 151 (1985).

${ }^{11}$ M. Marquillie and U. Ehrenstein, "On the onset of nonlinear oscillations in a separating boundary-layer flow," J. Fluid Mech. 490, 169 (2003).

${ }^{12} \mathrm{~B}$. Pier, "On the frequency selection of finite-amplitude vortex shedding in the cylinder wake," J. Fluid Mech. 458, 407 (2002).

${ }^{13} \mathrm{~V}$. Theofilis, S. Hein, and U. Dallmann, "On the origins of unsteadiness and three dimensionality in a laminar separation bubble," Philos. Trans. R. Soc. London, Ser. A 358, 3229 (2000).

${ }^{14}$ D. Barkley, M. Gomes, and D. H. Genderson, "Three dimensional instability in flow over a backward-facing step," J. Fluid Mech. 473, 167 (2002).

${ }^{15}$ F. Gallaire, M. Marquillie, and U. Ehrenstein, "Three-dimensional transverse instabilities in detached boundary layers," J. Fluid Mech. 571, 221 (2007).

${ }^{16}$ B. Wasistho, B. J. Geurts, and J. G. M. Kuerten, "Numerical simulation of separated boundary-layer flow,” J. Eng. Math. 32, 177 (1997).

${ }^{17} \mathrm{~V}$. Theofilis, "Advances in global linear instability of nonparallel and three-dimensional flows," Prog. Aerosp. Sci. 39, 249 (2003).

${ }^{18}$ C. Cossu and J.-M. Chomaz, "Global measures of local convective insta- 
bilities," Phys. Rev. Lett. 78, 4387 (1997).

${ }^{19}$ J.-M. Chomaz, "Global instabilities in spatially developing flows: Nonnormality and non linearity," Annu. Rev. Fluid Mech. 37, 357 (2005).

${ }^{20}$ U. Ehrenstein and F. Gallaire, "On two dimensional temporal modes in spatially evolving open flows: The flat-plate boundary layer," J. Fluid Mech. 536, 209 (2005).

${ }^{21}$ F. Alizard and J.-C. Robinet, "Spatially convective global modes in a boundary layer," Phys. Fluids 19, 114105 (2007).

${ }^{22}$ E. Åkervik, U. Ehrenstein, F. Gallaire, and D. S. Henningson, "Global two-dimensional stability measures of the flat plate boundary-layer flow," Eur. J. Mech. B/Fluids 27, 501 (2008).

${ }^{23}$ P. J. Schmid and D. Henningson, "On the stability of a falling liquid curtain," J. Fluid Mech. 463, 163 (2002).

${ }^{24}$ E. Åkervik, J. Hoepffner, U. Ehrenstein, and D. S. Henningson, "Optimal growth, model reduction and control in a separated boundary-layer flow using global eigenmodes," J. Fluid Mech. 579, 305 (2007).

${ }^{25}$ D. S. Henningson and E. Åkervik, "The use of global modes to understand transition and perform flow control," Phys. Fluids 20, 031302 (2008).

${ }^{26} \mathrm{U}$. Ehrenstein and F. Gallaire, "Global low-frequency oscillations in a separating boundary-layer flow," J. Fluid Mech. 614, 315 (2008).

${ }^{27}$ H. M. Blackburn, D. Barkley, and S. J. Sherwin, "Convective instability and transient growth in flow over a backward-facing step," J. Fluid Mech. 603, 271 (2008).

${ }^{28}$ O. Marquet, D. Sipp, J.-M. Chomaz, and L. Jacquin, "Amplifier and resonator dynamics of a low-Reynolds-number recirculation bubble in a global framework," J. Fluid Mech. 605, 429 (2008).

${ }^{29}$ P. J. Schmid, "Nonmodal stability theory," Annu. Rev. Fluid Mech. 39, 129 (2007).

${ }^{30}$ L. L. Pauley, P. Moin, and W. Reynolds, "The structure of two dimensional separation,” J. Fluid Mech. 220, 397 (1990).
${ }^{31}$ M. D. Ripley and L. L. Pauley, "The unsteady structure of twodimensional steady laminar separation," Phys. Fluids A 5, 3099 (1993).

${ }^{32}$ O. Daube, "Resolution of the 2D Navier-Stokes equations in velocityvorticity form by means of an influence matrix technique," J. Comput. Phys. 103, 402 (1992).

${ }^{33}$ R. Peyret, Spectral Methods for Incompressible Viscous Flow (Springer, New York, 2002).

${ }^{34}$ M. Pernice and F. W. Homer, "Nitsol: A Newton iterative solver for nonlinear systems," SIAM J. Sci. Comput. (USA) 19, 302 (1998).

${ }^{35}$ T. N. Phillips and G. W. Roberts, "The treatment of spurious pressure modes in spectral incompressible flow calculations," J. Comput. Phys. 105, 150 (1993).

${ }^{36}$ R. B. Lehoucq, D.C. Sorensen, and C. Yang, ARPACK Users' Guide: Solution of Large Scale Eigenvalue Problems with Implicitly Restarted Arnoldi Methods (SIAM, Philadelphia, 1998).

${ }^{37}$ P. J. Schmid and D. S. Henningson, Stability and Transition in Shear Flows (Springer, New York, 2001).

${ }^{38}$ K.-C. Toh and L. N. Trefethen, "Calculation of pseudospectra by the Arnoldi iteration," SIAM J. Sci. Comput. (USA) 17, 1 (1996).

${ }^{39}$ J. C. M. Lin and L. L. Pauley, "Low-Reynolds-number separation on an airfoil," AIAA J. 34, 1570 (1996).

${ }^{40}$ C. P. Häggmark, A. A. Bakchinov, and P. H. Alfredsson, "Experiments on a two-dimensional laminar separation bubble," Philos. Trans. R. Soc. London, Ser. B 358, 3193 (2000).

${ }^{41}$ L. L. Pauley, "Structure of local pressure-driven three-dimensional transient boundary-layer separation," AIAA J. 32, 997 (1994).

${ }^{42}$ L. L. Pauley, "Response of two-dimensional separation to threedimensional disturbances," ASME J. Fluids Eng. 116, 433 (1994).

${ }^{43}$ T.-R. Bewley and S. Liu, "Optimal and robust control and estimation of linear paths to transition," J. Fluid Mech. 365, 305 (1998). 